\title{
Differentiating gastrointestinal stromal tumors from gastric adenocarcinomas and normal mucosae using confocal Raman microspectroscopy
}

\author{
Chih-Wei Hsu \\ Chia-Chi Huang \\ Jeng-Horng Sheu \\ Chia-Wen Lin \\ Lien-Fu Lin \\ Jong-Shiaw Jin \\ Wenlung Chen
}




\title{
Differentiating gastrointestinal stromal tumors from gastric adenocarcinomas and normal mucosae using confocal Raman microspectroscopy
}

\author{
Chih-Wei Hsu, ${ }^{\text {a,b }}$ Chia-Chi Huang, ${ }^{c}$ Jeng-Horng Sheu, ${ }^{b}$ Chia-Wen Lin, ${ }^{d}$ Lien-Fu Lin, ${ }^{e}$ Jong-Shiaw Jin, ${ }^{f}$ and \\ Wenlung Chen ${ }^{b, *}$ \\ 'Tungs' Taichung MetroHarbor Hospital, Division of General Surgery, Department of Surgery, No. 699, Section 8 , \\ Taiwan Boulevard, Wuqi, Taichung 43503, Taiwan

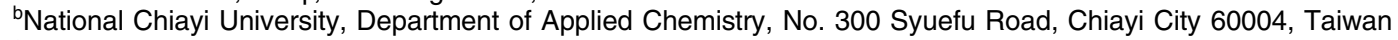 \\ 'National Chung Cheng University, Center of Nano Bio-Detection, No. 168, Section 1, University Road, Min-Hsiung Township, \\ Chia-yi County 621, Taiwan \\ dHungKuang University, Department of Applied Cosmetology, No. 34, Zhongqi Road, Shalu Township, Taichung County, Taiwan \\ 'Tungs' Taichung MetroHarbor Hospital, Division of Gastroenterology, Department of Internal Medicine, No. 699, Section 8, \\ Taiwan Boulevard, Wuqi, Taichung 43503, Taiwan \\ 'Tungs' Taichung MetroHarbor Hospital, Department of Pathology, No. 699, Section 8, Taiwan Boulevard, Wuqi, Taichung 43503, Taiwan
}

\begin{abstract}
Gastrointestinal stromal tumors (GISTs) are the most common mesenchymal neoplasms of the gastrointestinal tract, and gastric adenocarcinomas are a common cancer worldwide. To differentiate GISTs from adenocarcinomas is important because the surgical processes for both are different; the former excises the tumor with negative margins, while the latter requires radical gastrectomy with lymph node dissection. Endoscopy with biopsy is used to distinguish GISTs from adenocarcinomas; however, it may cause tumor bleeding in GISTs. We reported here the confocal Raman microspectroscopy as an effective tool to differentiate GISTs, adenocarcinomas, and normal mucosae. Of 119 patients enrolled in this study, 102 patients underwent gastrectomy (40 GISTs and 62 adenocarcinomas), and 17 patients with benign lesions were obtained as normal mucosae. Raman signals were integrated for $100 \mathrm{~s}$ for each spot on the specimen, and 5 to 10 spots, depending on the sample size, were chosen for each specimen. There were significant differences among those tissues as evidenced by different Raman signal responding to phospholipids and protein structures. The spectral data were further processed and analyzed by using principal component analysis. A two-dimensional plot demonstrated that GISTs, adenocarcinomas, and normal gastric mucosae could be effectively differentiated from each other. $\odot$ 2016 Society of Photo-Optical Instrumentation Engineers (SPIE) [DOI: 10.1117/1.JBO.21.7.075006]
\end{abstract}

Keywords: Raman spectroscopy; gastrointestinal stromal tumor; gastric adenocarcinoma.

Paper 150864RRR received Dec. 24, 2015; accepted for publication Jun. 16, 2016; published online Jul. 11, 2016.

\section{Introduction}

Gastrointestinal stromal tumors (GISTs) are the most common mesenchymal tumors of the gastrointestinal tract. ${ }^{1,2}$ They are derived from the interstitial cells of Cajal, which acts as a pacemaker for the gut. ${ }^{3-6}$ The stomach is the most common originating site of GISTs and accounts for $50 \%$ to $70 \%$ of all GISTs. ${ }^{1,7}$ GISTs are now considered as a potential malignancy, ${ }^{8-10}$ and even small, intramural lesions of the gastrointestinal tract may need resection. ${ }^{11}$ Varieties of the surgical procedure, including total gastrectomy, segmental or wedge resection, and lymph node dissection, have been approached to resect the tumor with negative margins. For GISTs, lymph node dissection is usually not required due to given the low incidence of nodal metastases. ${ }^{12}$ In contrast, the surgical procedure for gastric adenocarcinomas, accounting for $\sim 10 \%$ of cancers, ${ }^{13}$ is quite different from that for GISTs. Tis (carcinoma in situ: intraepithelial tumor without invasion of the lamina propria) or T1a (tumor invades lamina propria or muscularis mucosae) cancers limited to the mucosa may be candidates for endoscopic mucosal resection. T1b (tumor invades submucosa) to T3 (T2: tumor invades muscularis propria and T3: tumor penetrates

*Address all correspondence to: Wenlung Chen, E-mail: wlchen@mail.ncyu .edu.tw subserosal connective tissue without invasion of visceral peritoneum or adjacent structures) cancers should have adequate gastric resection to achieve negative microscopic margins, typically over $4 \mathrm{~cm}$ from the gross tumor. T4 (tumor invades serosa, visceral peritoneum, or adjacent structures) tumors require en bloc resection of the structures involved. Furthermore, gastric resection should include the regional lymphatics. ${ }^{12}$

Before the surgical treatments, gastric endoscopy is usually used for diagnosing gastric diseases, and any suspicious gastric ulceration is biopsied during the examination. The correct rate of diagnosing an existing gastric cancer in a single biopsy is $\sim 70 \%$, and it improves to about $98 \%$ as performing seven biopsies from the ulcer margin and base. ${ }^{14}$ Endoscopic ultrasonography (EUS) is currently the most effective tool for diagnosing gastric subepithelial tumors, including GISTs, on the basis of the depth of tumor and the layer of origin on the gastric wall. ${ }^{15,16}$ However, EUS alone does not provide an accurate diagnosis due to the morphological characteristics. ${ }^{15-18}$ Cytology may distinguish benign tissue from malignant lesions, whereas it is less helpful for determining their pathological types. ${ }^{18} \mathrm{~A}$ major concern of applying biopsy in GISTs is tumor bleeding and seeding because of their soft and fragile nature. ${ }^{19,20}$ Therefore, 
developing a rapid and effective method to differentiate GISTs from adenocarcinomas would be helpful for the recognition and treatment of gastric tumors.

Raman spectroscopy provides a noninvasive technique for molecular analysis and has been applied to study various tumors. ${ }^{21-32}$ By using laser excitation at wavelength of $532 \mathrm{~nm}$ coupled with a confocal microscope, the Raman microspectroscopy has been used successfully to diagnose breast cancer ${ }^{24}$ and brain tumors ${ }^{27}$. Zhou et al. ${ }^{27,29}$ reported that the Raman spectroscopy under 532-nm excitation may enhance the vibrational mode associated with certain bonds but other vibrational modes remain unaffected. This enhanced amide II, hemoglobin, metalloprotein, mitochondrial electron transport protein, and cytochrome c. These enhancements help to reveal key differences in the spectra from cancerous and normal brain tissues ${ }^{27}$. Raman spectra, to evaluate the surgical margin of breast $^{28}$ and gastrointestinal ${ }^{29}$ tissues, were available. Confocal Raman microspectroscopy was also available for molecular composition of specific skin structures analysis in vivo. ${ }^{30}$ Although Raman analysis of gastric adenocarcinoma has been reported, ${ }^{21,22,31,32}$ there remains a lack of information about Raman spectroscopic study on GISTs. In this report, confocal Raman microspectroscopy was employed to differentiate gastric adenocarcinomas, GISTs, and normal mucosae.

\section{Materials and Methods}

\subsection{Patients and Tissues}

In total, 119 patients were enrolled between 2008 and 2011. Of these, 102 patients underwent simple or radical gastrectomy for GISTs or adenocarcinomas, and the remaining 17 patients, underwent operations for other benign lesions, were obtained as the normal gastric mucosae. The formalin-fixed, paraffinembedded tissue specimens were prepared as slides with a $3-\mu \mathrm{m}$ thickness and were subjected to Raman measurement. Before Raman measurement, the slides were treated with a dewaxing procedure with hexane for $5 \mathrm{~min}$, ethanol for $3 \mathrm{~min}$, methanol for $1 \mathrm{~min}$, and then were dried with $\mathrm{N}_{2}$. The pathological diagnoses of these patients were reviewed by at least two experienced pathologists. Clinical data were retrospectively obtained from the patients' medical records.

\subsection{Ethics Statement}

The Institutional Review Board of Tungs' Taichung MetroHarbor Hospital approved the study (approval No. 100006). All included patients provided written informed consent before the collection of specimens and clinical information.

\subsection{Raman Microspectroscopic Measurement}

Confocal Raman microspectroscopy provides a platform for acquiring detailed Raman spectra from a small volume of specimens. Raman spectra of GIST, gastric adenocarcinoma, and normal gastric mucosa were obtained by using a confocal laser microRaman system (MploRA, Horiba Jobin-Yvon, France) with CCD detector. All Raman measurements were performed at room temperature. A Raman spectrometer with 532-nm laser excitation coupled with a confocal microscope (Olympus BX41, $\mathrm{NA}=0.9$ ) with $100 \times$ objective lens was used. The solid-state diode laser with $8 \mathrm{~mW}$ was used as an excitation source. The laser power was attenuated after passing through optical elements (mirrors and filters) of light path, which would not degrade the sample. The excitation light beam was directly shining on the surface of specimen, and the backscattered light was collected, passed through the entrance slit $(100-\mu \mathrm{m}$ wide), dispersed by a diffraction grating (1200 grooves $/ \mathrm{mm})$, and detected by an air-cooled CCD detector. The exposure time for each spot was $100 \mathrm{~s}$ (integration time $1 \mathrm{~s}$, accumulation: 100 scan), and 5 to 10 spots, depending on the sample size, were chosen for each specimen. The area was primarily diagnosed as a cancer abundant area by the pathologist. Raman spectra were produced over the Raman shift 500 to $3100 \mathrm{~cm}^{-1}$.

\subsection{Principal Component Analysis and Linear Discriminant Analysis}

The spectra results were processed and analyzed with the ArrayTrack data analysis and interpretation tool at the U.S. Food and Drug Administration National Center for Toxicological Research. ${ }^{33}$ Each spectrum from 500 to $3100 \mathrm{~cm}^{-1}$ was divided into 1022 segments and analyzed using principal component analysis (PCA) and linear discriminant analysis (LDA) to distinguish GISTs, adenocarcinomas, and normal mucosae. The first two principal components calculated (PC1 and PC2), which contained majority of the information, were plotted against each other for visualisation purposes. LDA with 20 -fold cross-validation was used to evaluate the accuracy, sensitivity, and specificity of the differential diagnosis by confocal Raman microspectroscopy.

PCA is a multivariate technique used to classify and reduce the dimensionality of the spectral data. Orthogonal linear combinations to transform the original data into uncorrelated variables are termed PCs. LDA is another data reduction technique. The first few PCs were selected for LDA. PCA uses the most information from the original data and LDA maximizes the intergroup differences and minimizes the intragroup differences. Therefore, the eigenvectors of PCA and LDA are different ${ }^{34,35}$. In 20-fold cross-validation, the original sample is randomly divided into 20 equal-sized subsamples. One of the 20 subsamples is retained as the validation data for testing the model, and the remaining subsamples are used as training data. The cross-validation process is then repeated 20 times, and the 20 results from the folds can then be averaged to produce a single estimation. Sensitivity (true positive rate) defined as measuring the proportion of positives that are correctly identified as such, and specificity (true negative rate) defined as measuring the proportion of negatives that are correctly identified as such. Receiver operating characteristic (ROC) curves were generated by successively changing the thresholds to determine correct and incorrect classifications for all subjects. The threshold of the sensitivity and specificity was defined as the maximum sum of the sensitivity and specificity (Youden index). ${ }^{34-36}$ The ROC curve was made by PC1 using SigmaPlot software version 10.0 (Systat Software Inc. San Jose, California).

\section{Results and Discussion}

\subsection{Spectra of Raman Microspectrospy}

Raman spectroscopy provides a lot of molecular information and is powerful in characterizing molecular structure. Varieties of Raman techniques, such as Fourier transform (FT)-Raman, resonance Raman, surface-enhanced Raman scattering, tipenhanced Raman, coherence antistoke Raman, and confocal Raman microscope, have been developed for biological and 
biomedical applications. Each Raman technique exhibits its unique preference in a certain usage. Confocal Raman microscope offers decisive advantages in contrast enhancement, rejection of stray light, and discrimination of a well-defined spatial region in a complex multiphase specimen. Confocal microscope provides an efficient way to obtain interference-free Raman spectra. The optical microscope focusing laser point onto a diffraction-limit spot on the specimen improves significantly in the lateral resolution and depth discrimination. Zhou et al. ${ }^{27}$ successfully demonstrated the usage of a confocal microRaman system with 532-nm excitation to study human brain cancer. The magic laser wavelength at $532 \mathrm{~nm}$ would cause resonance Raman scattering associated to vibration in the brain tissues. The strength of the resonance Raman signal excited by $532 \mathrm{~nm}$ is stronger than that by $732 \mathrm{~nm}$ because $532 \mathrm{~nm}$ falls in the special wing of the flavin fluorescence spectrum, and the background noise from the wing in Raman scattering is nearly gone. ${ }^{37}$ Raman scattering with excitation in the infrared wavelength such as $1064 \mathrm{~nm}$ may eliminate fluorescence interference; however, it is not suitable for a CCD detector due to a dramatic drop in the quantum efficiency at $1064 \mathrm{~nm}$. In contrast, coupling with the advances in the CCD detector, confocal Raman microcope provides a noninvasive, interference-free, less sample preparative, and spatially resolving method for studying the molecular composition of gastric tumors.

Figure 1 shows the microspectroscopic images of normal mucosae, GIST, and adenocarcinoma. The area was primarily diagnosed as a cancer abundant area by a pathologist. The arrow indicated the spot on the specimen exposing to the laser beam. It is difficult to differentiate those tissues based on the microscopic image (Fig. 1) without sample staining with hematoxylin and eosin (H\&E) stain. However, Raman profiles exhibited significant difference in those gastric tissues as shown in Fig. 2. Raman spectra of normal mucosae, GISTs, and gastric adenocarcinomas were demonstrated in Figs. 2(a), 2(b), and 2(c), respectively. Characteristic Raman peaks at $861,1004,1098$ to $1128,1240,1342,1442,1584$, and $1655 \mathrm{~cm}^{-1}$ were assigned to the $\mathrm{C}-\mathrm{C}$ stretching, $\mathrm{C}-\mathrm{C}$ symmetric stretching, $\mathrm{C}-\mathrm{N}$ stretching, $\mathrm{C}-\mathrm{N}$ stretching and $\mathrm{N}-\mathrm{H}$ bending, $\mathrm{CH}_{3} \mathrm{CH}_{2}$ wagging, $\mathrm{CH}_{2}$ and $\mathrm{CH}_{3}$ bending, $\mathrm{C}=\mathrm{C}$ bending, and $\mathrm{C}=\mathrm{O}$ stretching, respectively. ${ }^{38}$ These peaks represented for proline, phenylalanine, phospholipids, amide III, collagen, phospholipids, phenylalanine, and amide I, respectively. ${ }^{38}$ There are significant differences in the characteristic Raman bands and the relative Raman intensity. Spectral difference among the GIST, gastric adenocarcinoma, and normal gastric mucosa was observed in three segments: 1098 to $1128 \mathrm{~cm}^{-1}$, 1240 to $1342 \mathrm{~cm}^{-1}$, and 1584 to $1655 \mathrm{~cm}^{-1}$. For example, the Raman signal at $1098 \mathrm{~cm}^{-1}$ of adenocarcinoma splitted into two peaks of 1098 and $1128 \mathrm{~cm}^{-1}$ for the GISTs and normal mucosae. This suggests that the character of phospholipids of the adenocarcinomas is different from that of the normal mucosae and GISTs. The intensity ratio of Raman peaks at 1240 and $1342 \mathrm{~cm}^{-1}$ exhibited differently too. In the GIST, the intensity of the $1240 \mathrm{~cm}^{-1}$ peak was almost equal to that of the $1342 \mathrm{~cm}^{-1}$ peak; however, in the adenocarcinoma and normal mucosa, the intensity of the $1240 \mathrm{~cm}^{-1}$ peak was obviously higher than that of the $1342 \mathrm{~cm}^{-1}$ peak. This implies that the proportion of amide III and collagen in these three types of gastric tissue is different. Another difference is in the relative Raman intensity between 1584 and $1655 \mathrm{~cm}^{-1}$. GIST and normal mucosa tissue had higher Raman intensity at $1655 \mathrm{~cm}^{-1}$, (a)

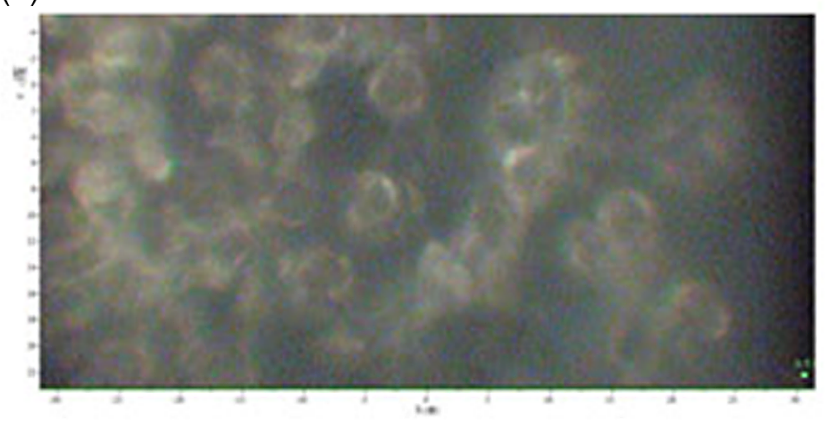

(b)

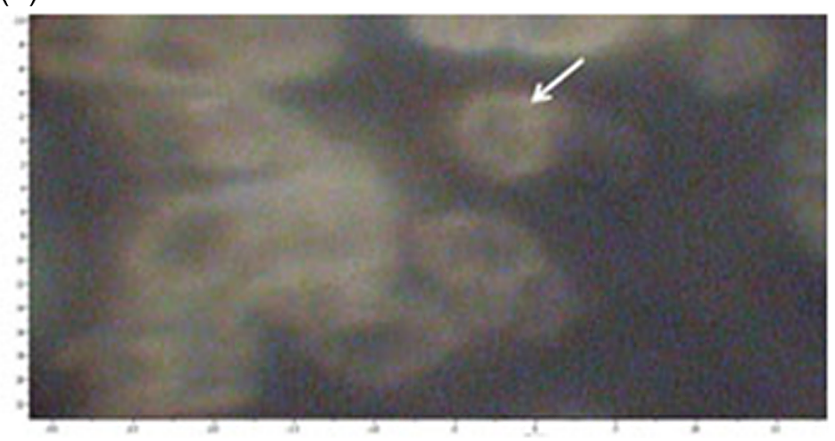

(c)

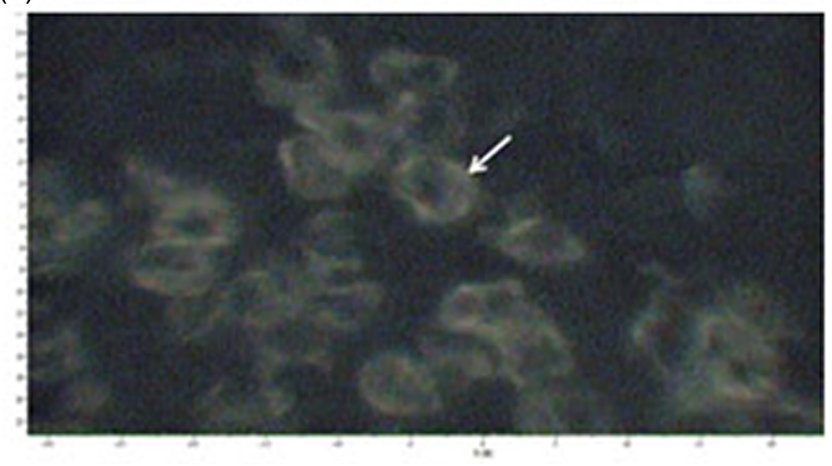

Fig. 1 Microspectroscopy images of gastric tissues (100× objective lens): (a) normal mucosae, (b) GIST, and (c) adenocarcinoma. The arrows pointed to the tumor cell.

but the $1584 \mathrm{~cm}^{-1}$ peak was absent in the adenocarcinoma spectrum. Difference in the intensity ratio of 1655 to $1584 \mathrm{~cm}^{-1}$ reflects to the different proportions of amide I to phenylalanine. Apparently, Raman spectra reflect faithfully to the biochemical and biomolecular characteristics.

\subsection{Principal Component Analysis and Linear Discriminant Analysis}

To enhance the application of Raman microspectrocopy in differentiating, the Raman spectral data were further analyzed by PCA and LDA. By using 20-fold cross-validation, the PC1 component accounted for the greatest Raman spectral variance of $90.838 \%$, and the PC2 component for $6.93 \%$. Plotting PC1 against PC2 (Fig. 3) showed the separation of GISTs, gastric adenocarcinomas, and normal gastric mucosae. For GISTs, the sensitivity, specificity, and accuracy were $100 \%, 97.50 \%$, and $99.16 \%$, respectively. The sensitivity, specificity, and accuracy for adenocarcinomas were $100 \%, 94.74 \%$, and $97.48 \%$, respectively, and for normal gastric mucosae, they were $99.02 \%$, 


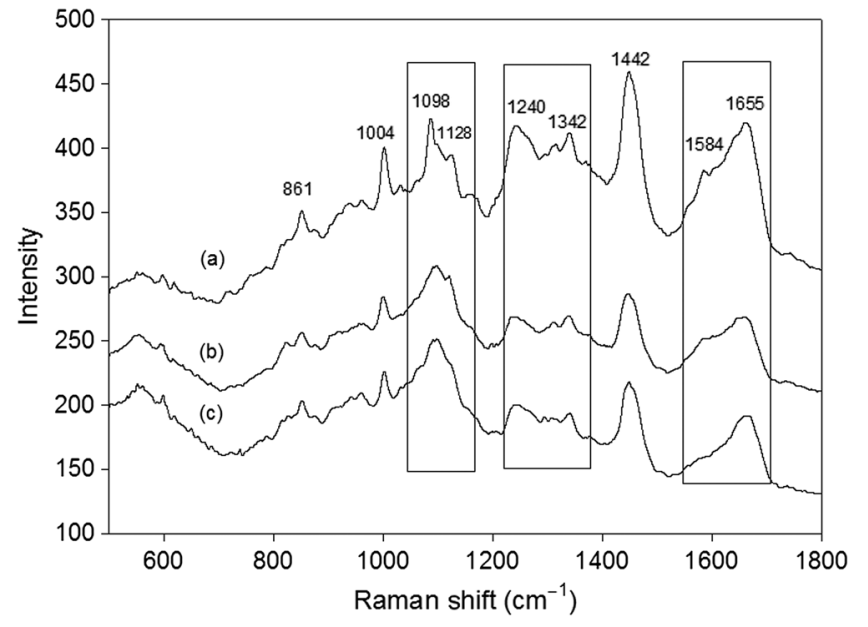

Fig. 2 Raman spectra, in the range from 500 to $1800 \mathrm{~cm}^{-1}$, of gastric tissues: (a) normal mucosa, (b) GIST, and (c) adenocarcinoma. Data acquisition conditions: excitation wavelength, $532 \mathrm{~nm}$; laser power, $8 \mathrm{~mW}$; and integration time $100 \mathrm{~s}$.

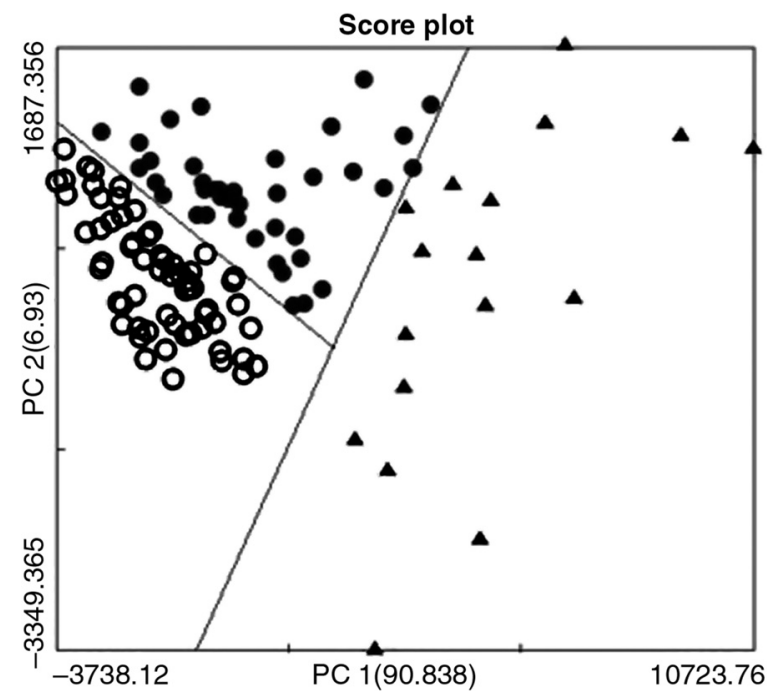

Fig. 3 PCA of the Raman spectral characteristics for gastric tissues. The first two principal components (PC1 and PC2) from the spectroscopy, which contained most information, were plotted against each other. The plot shows the separation between gastrointestinal stromal tumors, adenocarcinomas, and normal mucosae. (Closed circle: gastrointestinal stromal tumors; open circle: adenocarcinomas; and triangle: normal mucosae).

$94.12 \%$, and $98.32 \%$, respectively (Table 1). Clearly, each evaluated tissue sample fell within the correct gastric tissue region on the plot. In comparison, the diagnosis of GISTs with EUS-fine needle aspiration had an accuracy of $82 \%$ and a specificity of $100 \% .{ }^{39}$ Previously, a study evaluating intraoperative frozen sections to determine the margin of gastric cancer and to distinguish gastric adenocarcinomas from normal mucosae in the proximal end showed a sensitivity of $77.8 \%$, specificity of $100 \%$, and accuracy of $97 \%{ }^{40}$ Another study also showed that intraoperative frozen sections determined the distal margin in gastric adenocarcinoma with a sensitivity of $100 \%$, specificity of $100 \%$, and accuracy of $100 \%$, and the proximal margin with a sensitivity of $67 \%$, specificity of $100 \%$, and accuracy of $93 \%$, when performed by an expert gastrointestinal pathologist. ${ }^{41}$ In Fig. 4, we demonstrated the ROC curve of
Table 1 Performance report of sample classification using 20-fold cross-validation.

\begin{tabular}{lccc} 
Metric & GISTs & Adenocarcinomas & Normal \\
\hline Accuracy & $99.16 \%$ & $97.48 \%$ & $98.32 \%$ \\
Sensitivity & $100.00 \%$ & $100.00 \%$ & $99.02 \%$ \\
Specificity & $97.50 \%$ & $94.74 \%$ & $94.12 \%$ \\
Prevalence & $66.39 \%$ & $52.10 \%$ & $85.71 \%$ \\
Positive predictivity value & $98.75 \%$ & $95.38 \%$ & $99.02 \%$ \\
Negative predictivity value & $100.00 \%$ & $100.00 \%$ & $94.12 \%$ \\
False positive rate & $2.50 \%$ & $5.26 \%$ & $5.88 \%$ \\
False negative rate & $0.00 \%$ & $0.00 \%$ & $0.98 \%$ \\
Matthew's correlation & 0.98 & 0.95 & 0.93 \\
coefficient & & & \\
Area under ROC curve & 0.99 & 0.97 & 0.97 \\
\hline
\end{tabular}

gastric disease mucosa (GIST and adenocarcinoma) versus normal mucosa [Fig. 4(a)] and of GIST and normal mucosa versus adenocarcinoma [Fig. 4(b)], the area under the ROC curves is 0.99 and 0.88 , respectively. Apparently, the results obtained by confocal Raman microspectroscopic measurement are quite promising. Raman measurement requires a very small sample and only takes a few minutes without the need for tissue pretreatment such as immunohistochemical staining. By using confocal Raman microspectroscopy coupling with PCA analysis, we reported an effective method for differentiating GISTs from adenocarcinomas and normal gastric mucosae. It was also suggested to be applicable in in situ measurements as combined with endoscopy. ${ }^{42,43}$ To analyze the subgroups of patients with adenocarcinomas and GISTs, a larger sampling size including every subgroup of adenocarcinomas and GISTs would be necessary for further study of the gastric tumors.

Optical histopathology fast emerges as a potential tool in the diagnosis of various cancers. Fresh tissue is the best choice and serves as an ideal sample for optical histopathology. However, due to severe constraints associated with handling fresh tissues and inevitable problems in the time lapse between tissue excision and spectral analysis, tissue samples are usually preserved in formalin for postspectroscopic analysis. For example, in this study, we cannot investigate simultaneously all samples at one time, and it takes times to complete all Raman measurements of 119 samples, particularly as 5 to 10 spots of Raman measurement for each sample. Therefore, fixation of tissue samples is an inevitable process for protecting tissue from spoilage. The effect of formalin fixation on spectroscopic detection of normal and cancerous tissues has been reported. ${ }^{44-47} \mathrm{Xu}$ et al. ${ }^{44}$, using two-photon fluorescence spectroscopy to study mouse skeletal muscle, indicate that formalin is a better fixative than methanol for preserving samples in in vitro research. Huang et al. ${ }^{45}$ used a dispersive-type near-infrared Raman system with an excitation wavelength of $785 \mathrm{~nm}$ to study whether formalin fixation would affect the potential diagnostic ability for the detection of lung cancer. Although the overall intensities of Raman peaks decreased, the characteristic Raman peaks were found in both 

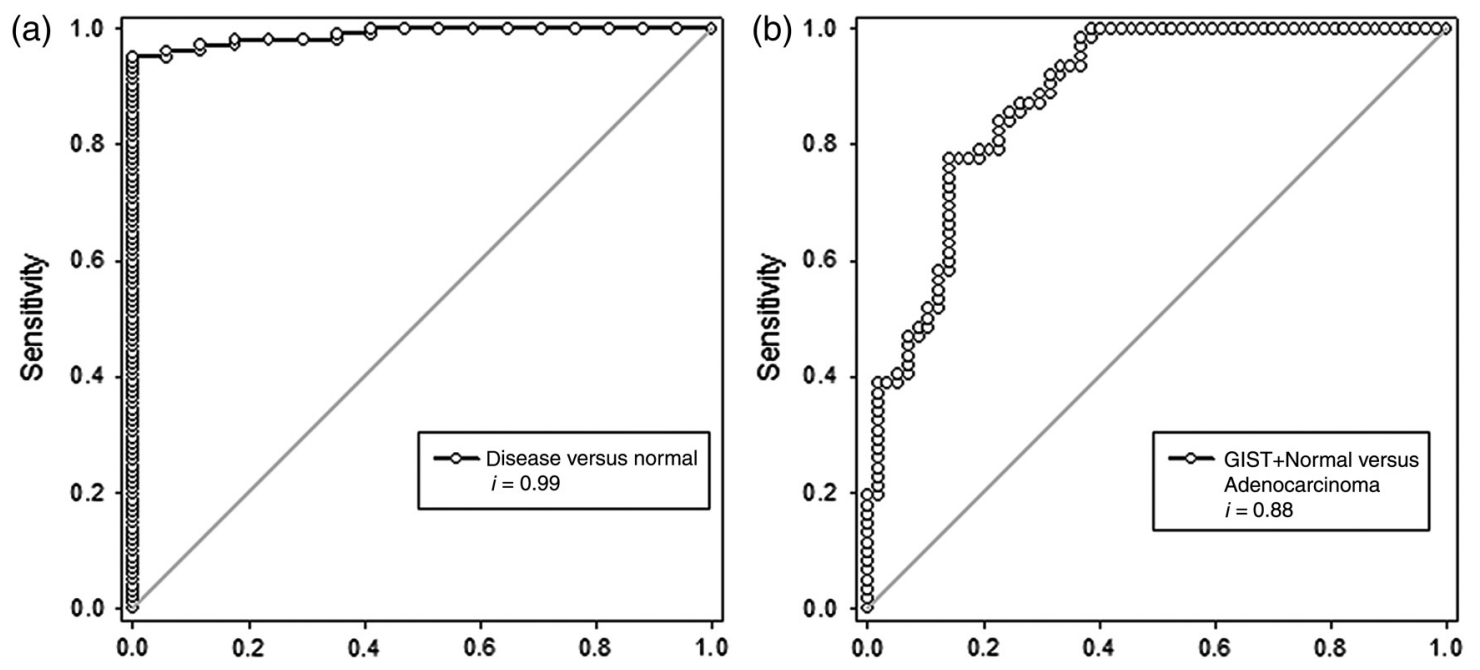

Fig. 4 (a) The ROC curve of disease (GIST and adenocarcinoma) versus normal mucosa, the area under the ROC curve is 0.99 . (b) The ROC curve of GIST and normal mucosa versus adenocarcinoma, the area under the ROC curve is 0.88 .

fresh and formalin-fixed tissues. In the earlier study on Raman image of eye lenses, ${ }^{46}$ it also showed that the overall intensity of Raman peaks decreased; however, there was no significant difference between the Raman profile of fresh and fixed lenses. Krishna et al. $^{47}$ also indicated feasibility of using formalinfixed tissues in optical histopathology, especially from Raman spectroscopy point of view. Resonance enhancement by using 532-nm excitation in Raman measurement as described by Alfano et al. ${ }^{24,29,37}$ may offset Raman signal attenuation caused by fixation of tissue. Hence, in this study, although fixation of the tissue may attenuate Raman intensity, Raman signals of various sample tissues (Fig. 2) are quite promising and differentiation of normal and cancerous tissues can be easily achieved. We successfully demonstrate that confocal Raman microspectroscopy is powerful to differentiate GISTs, adenocarcinomas, and normal gastric mucosae. To enhance the application of confocal Raman microspectroscopy in gastric tumors, a study combining endoscopy or EUS in situ would much help to further evaluate the characteristics of gastric tissues and may allow an intraoperative measurement.

\section{Conclusion}

In the process of carcinogenesis, the molecular structure of fundamental biomolecules, such as lipids, protein, carbohydrates, and nucleic acids, will encounter significant change, which ultimately results in a morphological variation. To date, the histopathological examination based on morphology of biopsy specimens is employed as a standard method to diagnose cancerous tissue. However, it is difficult to distinguish normal mucosae, GIST, and adenocarcinoma by microscopy without sample staining with H\&E, which is tedious and time-consuming. In contrast, Raman spectroscopy is rich in molecular information coming from the vibrational modes of biomolecular structure. It will offer a fast, simple, and direct method in detecting normal and abnormal tissues. Raman spectra, based on the investigation of 119 patients, clearly demonstrate that there are significant differences in phospholipids, amide III, and collagen among the GIST, gastric adenocarcinoma, and normal gastric mucosa. Coupling with PCA and LDA, confocal Raman microspectroscopy provides an effective method for the preoperative or intraoperative differentiation of GISTs, adenocarcinomas, and normal gastric mucosae, assisting in making rapid and appropriate decisions on tumor recognition and treatment in vitro. Combination of instrument for real time Raman measurement may make it possible for the in vivo study and clinical application.

\section{Acknowledgments}

The authors thank Professor Lai-Kwan Chau, Center of Nano Bio-Detection in National Chung Cheng University, for his assistance in the research.

\section{References}

1. M. Scarpa et al., "A systemic review on the clinical diagnosis of gastrointestinal stromal tumors," J. Surg. Oncol. 98(5), 384-392 (2008).

2. M. Miettinen et al., "Gastrointestinal stromal tumors: recent advances in understanding of their biology," Hum. Pathol. 30(10), 1213-1220 (1999).

3. G. H. Kim et al., "Digital image analysis of endoscopic ultrasonography is helpful in diagnosing gastric mesenchymal tumors," $B M C$ Gastroenterol. 14, 7 (2014).

4. I. Pidhorecky et al., "Gastrointestinal stromal tumors: current diagnosis, biologic behavior, and management," Ann. Surg. Oncol. 7(9), 705-712 (2000).

5. M. Sarlomo-Rikala et al., "CD117: a sensitive marker for gastrointestinal stromal tumors that is more specific than CD34," Mod. Pathol. 11(8), 728-734 (1998).

6. M. Miettinen, L. H. Sobin, and M. Sarlomo-Rikala, "Immunohistochemical spectrum of GISTs at different sites and their differential diagnosis with a reference to CD117 (KIT)," Mod. Pathol. 13(10), 1134-1142 (2000).

7. C. D. Fletcher et al., "Diagnosis of gastrointestinal stromal tumors: a consensus approach," Hum. Pathol. 33(5), 459-465 (2002).

8. M. Miettinen, L. H. Sobin, and J. Lasota, "Gastrointestinal stromal tumors of the stomach: a clinicopathologic, immunohistochemical, and molecular genetic study of 1765 cases with long-term followup," Am. J. Surg. Pathol. 29(1), 52-68 (2005).

9. M. Bruno et al., "The natural history of gastrointestinal subepithelial tumors arising from muscularis propria: an endoscopic ultrasound survey," J. Clin. Gastroenterol. 43(9), 821-825 (2009).

10. C. D. Fletcher et al., "Diagnosis of gastrointestinal stromal tumors: a consensus approach," Int. J. Surg. Pathol. 10(2), 81-89 (2002). 
11. J. Y. Blay et al., "Consensus meeting for the management of gastrointestinal stromal tumors. Report of the GIST Consensus Conference of 20-21 March 2004, under the auspices of ESMO," Ann. Oncol. 16(4), 566-578 (2005).

12. National Comprehensive Cancer Network, "Clinical practice guidelines in oncology," version 3.2015 (2015), http://www.nccn.org/ professionals/physician_gls/f_guidelines.asp

13. J. Ferlay et al., Globocan 2002: Cancer Incidence, Mortality, and Prevalence Worldwide, IARC Press, Lyon (2004).

14. D. Y. Graham et al., "Prospective evaluation of biopsy number in the diagnosis of esophageal and gastric carcinoma," Gastroenterology 82(2), 228-231 (1982).

15. J. H. Kim et al., "A feasible modified biopsy method for tissue diagnosis of gastric subepithelial tumors," World J. Gastroenterol. 19(29), 47524757 (2013).

16. M. R. Vander Noot, III et al., "Diagnosis of gastrointestinal tract lesions by endoscopic ultrasound-guided fine-needle aspiration biopsy," Cancer 102(3), 157-163 (2004).

17. J. H. Hwang, S. D. Rulyak, and M. B. Kimmey, "American Gastroenterological Association Institute technical review on the management of gastric subepithelial masses," Gastroenterology 130(7), 2217-2228 (2006).

18. C. Karaca et al., "Accuracy of EUS in the evaluation of small gastric subepithelial lesions," Gastrointest. Endosc. 71(4), 722-727 (2010).

19. J. H. Lee et al., "Clinical impaction of EUS-guided Trucut biopsy on decision making for patients with gastric subepithelial tumors $\geq 2 \mathrm{~cm}$ in diameter," Gastrointest. Endosc. 74(5), 1010-1018 (2011).

20. G. D. Demetri et al., "NCCN Task Force report: update on the management of patients with gastrointestinal stromal tumors," J. Natl. Compr. Cancer Network 8(Suppl. 2), S1-44 (2010).

21. A. G. Shen et al., "Screening of gastric carcinoma cells in the human malignant gastric mucosa by confocal Raman microspectroscopy," Vib. Spectrosc. 37(2), 225-231 (2005).

22. Y. Hu et al., "Classification of normal and malignant human gastric mucosa tissue with confocal Raman microspectroscopy and wavelet analysis," Spectrochim. Acta A Mol. Biomol. Spectrosc. 69(2), 378382 (2008).

23. R. R. Alfano et al., "Method for determining if a tissue is a malignant tumor tissue, a benign tumor tissue, or a normal or benign tissue using Raman spectroscopy," U.S. Patent No. 5,261,410 (1993).

24. C.-H. Liu et al., "Resonance Raman and Raman spectroscopy for breast cancer detection," Technol. Cancer Res. Treat. 12(4), 371-382 (2013).

25. K. E. Shafer-Peltier et al., "Raman microspectroscopic model of human breast tissue: implications for breast cancer diagnosis in vivo," J. Raman Spectrosc. 33(7), 552-563 (2002).

26. A. S. Haka et al., "Diagnosing breast cancer using Raman spectroscopy: prospective analysis," J. Biomed. Opt. 14(5), 054023 (2009).

27. Y. Zhou et al., "Human brain cancer studied by resonance Raman spectroscopy," J. Biomed. Opt. 17(11), 116021 (2012).

28. A. S. Haka et al., "In vivo margin assessment during partial mastectomy breast surgery using Raman spectroscopy," Cancer Res. 66(6), 33173322 (2006).

29. Y. Zhou et al., "Tumor margin detection using optical biopsy techniques," Proc. SPIE 8940, 894014 (2014).

30. P. J. Caspers, G. W. Lucassen, and G. J. Puppels, "Combined in vivo confocal Raman spectroscopy and confocal microscopy of human skin," Biophys. J. 85(1), 572-580 (2003).
31. S. K. Teh et al., "Diagnostic potential of near-infrared Raman spectroscopy in the stomach: differentiating dysplasia from normal tissue," $B r . J$. Cancer 98(2), 457-465 (2008).

32. T. Kawabata et al., "Optical diagnosis of gastric cancer using nearinfrared multichannel Raman spectroscopy with a 1064-nm excitation wavelength," J. Gastroenterol. 43(4), 283-290 (2008).

33. W. Tong et al., "ArrayTrack ${ }^{\mathrm{TM}}$-Supporting toxicogenomic research at the FDA's National Center for Toxicological Research," Environ. Health Perspect. 111(15), 1819-1826 (2003).

34. P. N. Belhumeru, J. P. Hespanha, and D. J. Kriegman, "Eigenfaces vs. fisherfaces: recognition using class specific linear projection," IEEE Trans. Pattern Anal. Mach. Intell. 19(7), 711-720 (1997).

35. L. A. Austin, S. Osseiran, and C. L. Evans, "Raman technologies in cancer diagnostics," Analyst 141(2), 476-503 (2016).

36. I. W. Schie and T. Huser, "Method and applications of Raman microspectroscopy to single-cell analysis," Appl. Spectrosc. 67(8), 813-828 (2013).

37. J. Murphy, "Spectroscopy heading toward 'fantastic voyage': a Q\&A with Robert Alfano and Lingyan Shi," Photonic Spectra 50(2), 5659 (2016).

38. Z. Huang et al., "Near-infrared Raman spectroscopy for optical diagnosis of lung cancer," Int. J. Cancer 107(6), 1047-1052 (2003).

39. R. R. Watson et al., "Yield and performance characteristics of endoscopic ultrasound-guided fine needle aspiration for diagnosing upper GI tract stromal tumors," Dig. Dis. Sci. 56(6), 1757-1762 (2011).

40. J. G. Shen et al., "Intraoperative frozen section margin evaluation in gastric cancer of the cardia surgery," Hepatogastroenterology 53(72), 976-978 (2006).

41. J. Spicer et al., "Diagnostic accuracy and utility of intraoperative microscopic margin analysis of gastric and esophageal adenocarcinoma," Ann. Surg. Oncol. 21(8), 2580-2586 (2014).

42. Z. Huang et al., "In vivo detection of epithelial neoplasia in the stomach using image-guided Raman endoscopy," Biosens. Bioelectron. 26(2), 383-389 (2010)

43. M. S. Bergholt et al., "Combining near-infrared-excited autofluorescence and Raman spectroscopy improves in vivo diagnosis of gastric cancer," Biosens. Bioelectron. 26(10), 4104-4110 (2011).

44. M. G. Xu et al., "Effect of handling and fixation processes on fluorescence spectroscopy of mouse skeletal muscles under two-photon excitation," Appl. Opt. 39(34), 6312-6317 (2000).

45. Z. Huang et al., "Effect of formalin fixation on the near-infrared Raman spectroscopy of normal and cancerous human bronchial tissues," Int. J. Oncol. 23(3), 649-655 (2003).

46. W. Chen, "Raman spectroscopic/imaging studies of eye lenses," thesis, Georgia Tech, Chap VII, pp. 141-151 (1992).

47. C. M. Krishna et al., "Vibrational spectroscopy studies of formalin-fixed cervix tissues," Biopolymers 85(3), 214-221 (2007).

Wenlung Chen is a professor in the Department of Applied Chemistry at the National Chiayi University, Taiwan. He received his $\mathrm{PhD}$ in chemistry from Georgia Institute of Technology. His current research interests include (I) purification, identification, enzyme activity, and structural characterization of functional protein (II) developing Raman techniques (conventional Raman, FT-Raman, resonance Raman, surface enhanced Raman scattering, and Raman imaging) in biological and biomedical applications.

Biographies for the other authors are not available. 